\title{
Application of Ad Hoc Technology on Fire Rescue Wireless Communications
}

\author{
ZHANG Shixing \\ Department of Fire Engineering \\ Chinese people's Armed Police Force Academy \\ Langfang, Hebei, China \\ e-mail: zs_xing@sina.com
}

\begin{abstract}
Ad Hoc networks is a new type of wireless, multihop and centerless distributed control network, and the network infrastructure is not required. Ad Hoc has the features of strong self-organizing and anti-destroying ability. In the article, the characteristics of Ad Hoc network was introduced, the structure was studied, and the application of Ad Hoc network technology on fire rescue wireless communications was discussed.
\end{abstract}

Keywords-Ad Hoc; fire rescue; wireless communication

\section{INTRODUCTION}

Communication is the important basis and supporting conditions for fire rescue command, and the timely, effective and reliable communication support is the key to insuring the success of rescue. After a fire disaster, the fixed communications network infrastructure may all be damaged or fail to work in the area, then, it is particularly important to set up temporary wireless broadband networks. The realtime dynamic information of fire scene is helpful to the fire commander to make decision properly and timely.

Ad Hoc network is a self-organizing network technology which independents of any fixed network infrastructure. Ad Hoc network can quickly lay and provide network communication support for fire rescue timely.

\section{AD HOC NETWORK FEATURES}

Wireless Ad Hoc network is constituted by a group of wireless mobile nodes, it is multi-hop, temporary, noninfrastructure supporting and centerless. Mobile terminal in the network has the function of routing and packet forwarding. An network topology can be composed by the wireless connection. The communication between nodes may be transmitted through multiple intermediate nodes, that is multi-hop, and it is the fundamental difference between Ad Hoc network and other mobile network ${ }^{[1]}$. By the coordination between layered network protocols and distributed algorithm, the network can realize automatic organization and operation. Figure 1 is a typical basic structure of Ad Hoc network.

In a general way, Ad Hoc network has four features, which are centerless, self-organization, multihop routing and dynamic topology.

Centerless is the most obvious feature of Ad Hoc network. There is no center in Ad Hoc network, and all access nodes in the network are equal. Any node can join or leave the network freely, and the failure of any node will not affect the normal operation of the network. It provides convenience for the network communication among the fire trucks.

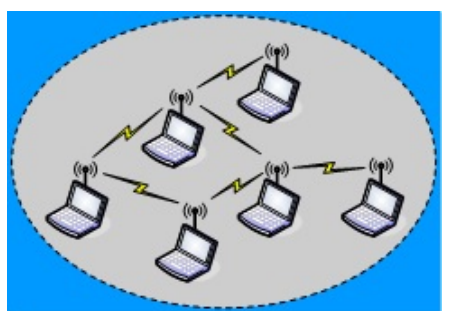

Figure 1. Typical basic structure of Ad Hoc network

Self-organization is another feature of Ad Hoc network. The layout of the network needn't relying on any of the network facilities. As long as there are more than two computers supporting Ad Hoc, a wireless connection can be established. Ad Hoc technology is very suitable for temporary network organization without relying on router or reticle. Two computers supporting Ad Hoc can immediately set up a network, and transfer informations mutually ${ }^{[2]}$.

In Ad Hoc network, if the distance exceeds the signal coverage, then the information can be transmitted by the module of intermediate node. So the multihop routing can be completed by the ordinary network nodes in Ad Hoc network.

The structure of Ad Hoc network is dynamic. When there is any computer joining or leaving the network, the structure of the network will make a dynamic change. The maximum speed of the Ad Hoc network can reach $300 \mathrm{~km} / \mathrm{h}$, and the switching delay time is less than $100 \mathrm{~ms}$. Thus, when fire truck installed Ad Hoc network travells to fire scene, the communications of video, voice and other data can be performed without interruption. At high speed condition, the throughput between the moving vehicle and other fixed Ad Hoc network nodes can reach $15 \mathrm{Mbps}$, which can fully meet the demand of high bandwidth in fire rescue ${ }^{[4]}$.

\section{APPLICATION OF AD HOC NETWORK IN FIRE RESCUE}

Based on the characteristics of Ad Hoc, the wireless network technology can be applied to fire rescue wireless communication network. When Ad Hoc network terminals are installed on wearable computers of fire fighters and car computer as network nodes, a temporary network is formed in fire site, and a wireless broadband data net is builted. 


\section{A. Node Structure Of Fire Fighting Unit}

Ad Hoc network node not only has the normal function of mobile device, but also has the ability to forward packets, that is, the node has the router function. Therefore, the nodes can be divided into three parts: host, router, and station. The host part completes the ordinary mobile terminal function, including human-machine interface, data processing and other application. The router part is responsible for maintaining the network topology structure and routing information to complete the packet forwarding. The station part provides wireless channel support for $t$ information ransmission.

From the physical structure point, the nodes can be divided into the following categories (as shown in Figure 2): single host single station, single host multi-station, multihost single station and multi-host multi-station ${ }^{[3]}$. Fire fighters nodes generally use the simple structure of single host single station as shown in Figure 2 (a). Fire communication command vehicle nodes may include more than one host, which using the structure of Figure 2 (c) and realizing multiple hosts sharing one or more stations. The multi-station not only can be used to build a overlay network, but also can be used as gateway node to interconnect multiple Ad Hoc network. General fire trucks can adopt the structure shown in Figure 2 (b).
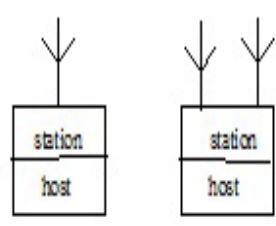

(b)

(a)

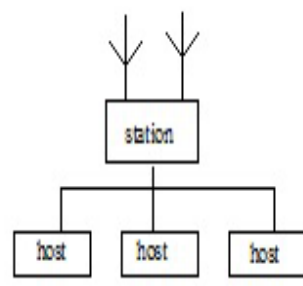

(c)
Figure 2 structure of Ad Hoc network nodes

((a) single host single station;(b) single host multi-station; (c)multi-host single/multi-station)

\section{B. Structure of Fire Rescue Communication Network}

In general, Ad Hoc network have two kinds of structure: plane structure and hierarchical structure.

In small scale fire rescue, which only fire squadron involved, the number of the fire fighters and the fire trucks is small, and the coverage area is limited, the chain of command is simple, so the plane structure can be used to organize the network. The positions of all nodes are equal in plane structure, and this kind of structure can also be called structure of equality( as shown in Figure 3). Plane structure network is relatively simple, all nodes in the network are completely peer-to-peer, in principle, there isn't any bottleneck, and it is robust, while the drawback of plane structure is the poor expandability. To each node, the route to reach all other nodes needs to know. Thus, a lot of control messages are required to maintain these dynamic changing routing information.

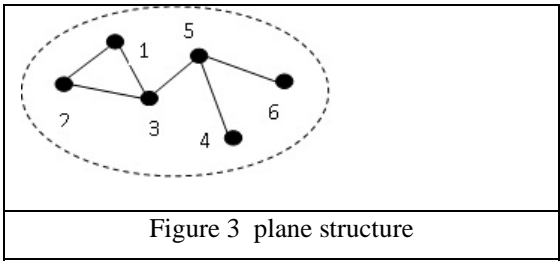

In big scale fire site, more fire teams attend,and the teams may come from other cities or other provinces.In this case, hierarchically structured network can be used and the network is divided into clusters. Each cluster consists of a cluster head and some cluster members. These cluster heads form a high-level network. The high-level network can also be divided into clusters, and a higher-level network is formed again until to the highest. In actual network, fire communication command vehicle can be used as cluster head. Cluster head node is responsible for transmittion of data among the cluster members. Cluster head can be prespecified, or be automatically elected by algorithm..

At the same time, hierarchical structure network can be divided into single-frequency hierar and multi-frequency hierar. In single frequency hierarchical network (as shown in Figure 4), all the nodes use the same frequency. In order to achieve communication between cluster heads, the support of gateway node is requisite (the node belongs to two clusters). In multi-frequency hierarchical network(as shown in Figure 5), different levels use different communication frequency. The communication range of lower nodes are smaller, and the range of higher nodes are larger ${ }^{[5]}$.
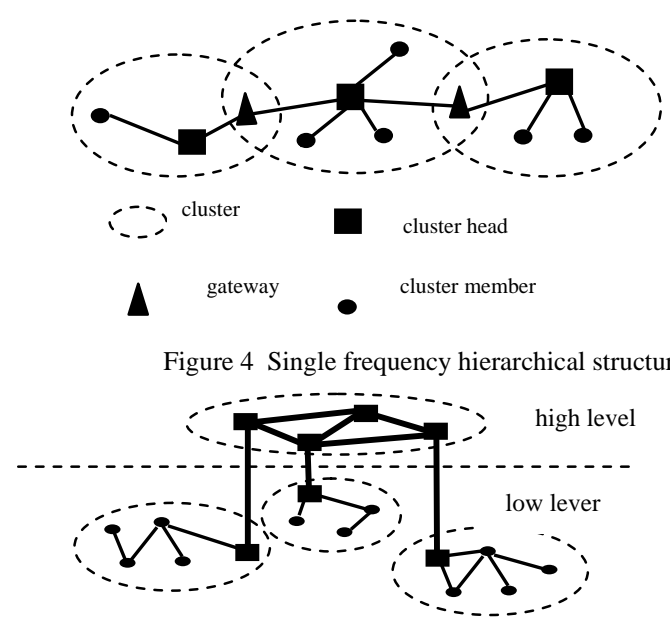

Figure 5 Multi-frequency hierarchical structure

Based on the temporary wireless network built by Ad Hoc technology, fire communication command vehicle can dynamically gather real-time informations of fire site, and transmit uninterruptedly to fire command center. The informations include various forms of fire fighting 
commands (graph, text, voice and etc), the real-time images of fire site and the state of the fire trucks. Fire trucks can join or leave the temporary network at any time, and it will not affect the data transmission of other vehicles with the fire command center. According to the informations received, the commander may carry on "on-scence command".

\section{CONCLUSION}

The application of Ad Hoc network technology in fire rescue solves the problems of traditional communication. Ad Hoc network realized the integration of collection, processing, communication and application of fire scence informations such as photos, text and other data, which provides strong technical and data supports to fire monitoring and fire remote decision.it is helpful to improve the efficiency of fire rescue and the ability to resist emergency fire disaster.

\section{REFERENCES}

[1] Zheng shaoren, “Ad Hoc Network Technique”,Beijing: Posts \& Telecom press,2005

[2] Wu gongyi, “Computer Network”, Tsinghua University Press,2007

[3] Zhu tingting and Zhao lin, “Application of Ad Hoc on Military Wireless Communication”, Ship Electronic Engineering,2006 (6)

[4] Cheng shaowei and Fan yufeng,Wireless Communication Networking Technology of the Fire Rescue,Fire Technology and Products Information, 2007 (4)

[5] He chengyong and Li fangwei,Key Technology of Ad Hocnetwork, Telecommunications Information,2008（2） 